\title{
Intelligent Pet Station Based on Internet of things
}

\author{
Ji Yu \\ North China Electric Power University, 2 Beinong Road, Huilongguan Town, Changping District, Beijing, \\ China
}

Keywords: Internet of Things; automation; care; pet station

\begin{abstract}
With the development of society, the demand of pet industry develops rapidly. More and more pets have become an important member of the family, and their owners take care of them as if they were children. With the continuous improvement of economic level and the rapid development of tourism, the desire of pet owners to travel is becoming more and more urgent. However, due to the restrictions of transportation and accommodation in China, it is very difficult for pets to go out with their owners, so they have to be forced to foster care. However, the standard of pet foster care and the management of epidemic prevention are very difficult, the pet disease or death and other problems not only are the issue of attribution of responsibility, and it is difficult to reach agreement on compensation.
\end{abstract}

Smart pet station came into being, which can take care of pets when owners leave. It can be used in personal family to take care of the pet when the owner leaves; it can be used in the pet hospital to manage foster pets, and for owners to check pet situation and living environment at any time. The main functions include four aspects.

1) Intelligent monitoring system: owners can view their pets and their living environment through mobile phone APP anytime and anywhere.

2) Automatic feeding device: set the time and times of feeding every day before using, and pet station will deliver proper amount of water and food to cage on time.

3) Timing release function: pet station will open cage door and release pet according to the time set by owner.

4) Daily cleaning function: every 24 hours pet station can change cage bottom materials to provide a clean and comfortable environment for pets.

\section{Introduction}

\subsection{Research Background}

With the development of social economy and the acceleration of urbanization process, people's spiritual life and material life are improving constantly, the pace of social aging is quickening, the popularization of one-child family and dink family, etc., and the leisure of the people. Consumption and emotional sustenance have also developed in a variety of ways. Pets have entered ordinary people's homes, keeping pets has become an essential pastime for families. With the increasing demand for pets, a new industry-pet industry is emerging. The emergence of this industry is the result of the improvement of people's living standard and the improvement of consumption level. It is also a social phenomenon under the condition of reform and opening up in China. However, due to the rapid pace of life, many people will neglect the care of pets, so pet feeding machine has become an essential tool for pet lovers.

Since the eighties of last century, the remote information transmission technology using the network has started, and with the continuous development of the network technology, the remote control technology has been gradually promoted. Make remote control technology become more and more practical and feasible ${ }^{[1]}$. Aiming at the problem that traditional pet feeding machine needs manual close control, this system takes the intelligent remote control system of pet feeding machine as the research object, on the basis of fully understanding the current situation of pet feeding 
machine at home and abroad. Adopting the advanced GPRS data packet wireless communication technology, the intelligent pet post system is designed and developed.

\subsection{Research Significance}

The pet market still has huge growth potential, especially in emerging markets. Pet health products and food additives will grow strongly by 12 percent and 8.4 percent a year, according to a survey of the Chinese pet products market in 2017. With the rapid development of our economy, the future of our pet industry is bright.

The number of pet owners in China is increasing and the number of pet owners in China is expected to increase explosively in the next decade. ${ }^{[2]}$ However, pets and work, leisure cannot be both, this is the pain of most pet lovers; home has a "baby", cannot safely go out, as if long imprisoned in a city. Pet foster care arises at the historic moment, the interaction between owner and pet becomes the focus. Intelligent pet cage is modified and upgraded on the basis of traditional pet cage, and the technology such as Internet of things is applied to take care of pets when the owner leaves. Avoid the trouble of pet foster care and disputes, to meet the growing love needs of users. To achieve the intelligent pet care, automation.

\subsection{Research Status}

Pet feeding machines are the first choice of pet feeding tools for pet lovers today, and realistic research has found that, despite having pet feeding machines, people still worry about whether they are working on time. Pet eating and other problems, some people may even use only a little rest time to drive home to take care of their pets, which is a lot of work for pet lovers. In view of the fact that the common feeding machine can only eat at a specified time and the user needs to observe the status of pet feeding at close range, it is necessary to study a remote control system for the pet feeding machine. In recent years, with the continuous development of information technology, people have gradually entered an information society, whether it is the current popular 4G network or popular short message service is to better provide people with wireless communication applications, and how to use this information to better serve the public has become an important topic of today's research. This paper studies the application of remote control technology to people's lives, and puts forward an intelligent remote control system of pet feeding machine, which is based on GPRS network transmission technology.

The basic service of GPRS network, short message service, is used for remote communication, which enables users to remotely control the corresponding functions of pet feeding machine. The system not only allows users to use $G$ at any time Any place covered by the PRS network can remotely control the pet feeding machine to feed the pet, and can also send the current pet feeding machine food quantity and water quantity to the user in the form of short message, which is convenient and quick. Can solve the user's concern about the pet's current food status. Therefore, the system has a very good practical value.

\section{Related Technology Research}

\subsection{Web technology}

Web technology supports the integration and development of cross-platform aggregation applications, as well as the universality of multi-device terminals (such as desktop computers, portable computers, mobile devices, tablets, gaming devices, etc.) It makes it the best candidate for a universal application integration platform. The mature technology system and the characteristics of open and simple development make it have established a perfect ecosystem on the Internet, and the network based on it has become the trend of network convergence in the future. Technology as the core technology in the Internet will become one of the important technical means in the process of network convergence.

Dr Web wrote on his blog: "the goal of the World wide Web is to create a place where information can be shared. We should have a place where everyone can contribute and write. ". Web 
is a system of many hypertext documents that are linked to each other and provide a mechanism for content negotiation in the protocol. Clients can choose the best possible from a variety of presentation formats. Because Web technology has the characteristics of distributed, loosely symmetric, extensible, easy to realize the aggregation of resources and services, can support the information exchange at semantic level, and the short users of low development cycle can participate in innovation and low cost, etc. Internet of things application architecture based on Web technology is becoming an important choice of wireless, mobile and cross-platform devices.

\subsection{GPRS}

GPRS is a kind of mobile packet data service developed on the basis of existing GSM mobile communication system, which can provide end-to-end. The technical advantage of wide area wireless IP connection. GPRS mainly lies in the realization of the best combination of user data and wireless network resources and the transparent transmission of IP protocol. The theoretical value of data transmission rate can reach 171 . $2 \mathrm{~kb} / \mathrm{s}$, which is more than ten times of the existing GSM network, can achieve higher data transmission on the basis of the original GSM network because it is based on the multiplexing mechanism of multiple GSM slots, and different network users share a set of channels. By means of multiplexing mechanism, channel resources can be utilized more efficiently. GPRS can support two main packet data network protocols: IP and X.25. They are always online, high data transmission rate and high spectrum efficiency. According to the characteristics of traffic charging and fast login, it is especially suitable for intermittent, sudden or frequent, small amount of data transmission, but also for occasional mass data transmission. ${ }^{[3]}$

With the rapid development of high and new technology, such as electronics and computer, the automation degree of electric power system, industrial production, traffic management and communication system is more and more high. The high degree of automation makes the process of production, monitoring and management more convenient and humanized. Some systems work in harsh environment, such as high pressure, high temperature, high altitude and underground. It is very difficult to monitor these systems, which requires a lot of manpower and financial resources. The emergence of remote monitoring technology has solved this problem well. This paper presents a remote monitoring system based on General Packet Radio Service (GPRS) technology. The remote monitoring system based on GPRS technology has the characteristics of wide application range, high reliability and low cost. It can be applied to all kinds of remote monitoring systems, such as power equipment remote monitoring, motor speed regulation system and so on.

\section{System Architecture}

\subsection{Monitoring Function}

The system has the function of monitoring and nursing, the mobile terminal and the pet care device are connected through the wireless network. ${ }^{[4]}$ The intelligent care device can collect the pet in the cage, send it to the central processor of the intelligent care device, and send the pet information to the mobile terminal by the central processor through the network cloud, and the owner receives the distance. The process pet information can be viewed on the mobile App.

\subsection{Feeding function}

First, the system automatically detects whether the current food and water containers have reached the full value or not. Then, the user sets the number of times a day to feed and the feeding time each time according to the demand. When the first set time is reached, the controller is operated by an operational amplifier through a weighing sensor under the food tray and the water container plate. If the current value is determined to be less than the maximum value, the controller controls the solid state relay to open the food tank or the corresponding switch device under the sink. For food or water, when the sampling value is near the maximum, stop the drop, thus completing the process, and control the miniature electric push rod and the miniature solenoid valve switch device-solid state relay, And the power supply to the whole system. 


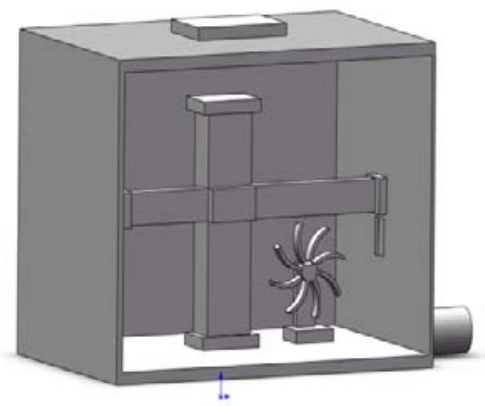

Fig.1 Feeding device

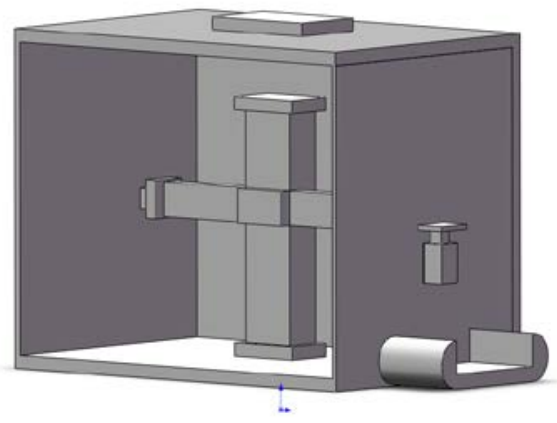

Fig.2 Feeding device

\subsection{Cleaning function}

The pet post station device also includes a cleaning device including a waste collector disposed at a corner of the cage. The excreta collector is replaced once every 24 or 48 hours depending on the owner's setting.

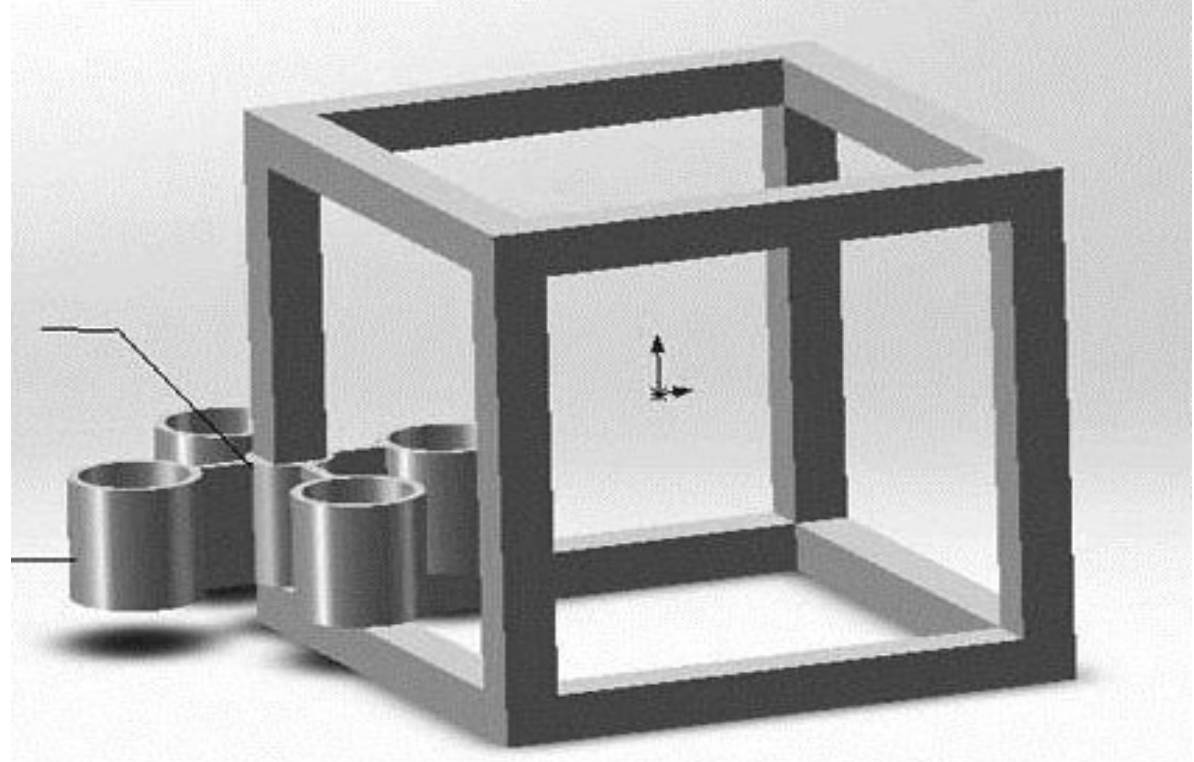

Fig.3 Cleaning device

\subsection{Timed-release}

The pet post station has an electromagnetic switch; the electromagnetic switch also sets the infrared sensor. When the infrared sensor receives the pet information, the electromagnetic switch controls the cage door of the pet cage closed.

\section{Summary}

In today's society, people's work and study are very busy, especially when people go on business trips or travel on vacation, they often fail to take good care of all kinds of pets at home in time, especially the diet of pets. It has often become one of the problems that beset people. The successful realization of the new intelligent pet post completely fills the gap of intelligent pet feeding in the domestic market, and in the aspects of design idea, practical application value and man-machine interaction of the system, the system is far superior to similar products on the international market, and the system is proved to be a very successful design. It not only brings economic benefits, but 
also plays a positive role in home intellectualization. ${ }^{[5]}$ While improving people's life efficiency and fun, it also reduces the worries for people's travel.

\section{References}

[1] Petri net-based context modeling for context-aware systems [J]. Seungwok Han, Hee Yong Youn. Artificial Intelligence Review. 2012 (1)

[2] Context modelling and a context-aware framework for pervasive service creation: A model-driven approach [J]. Achilleas Achilleos, Kun Yang, Nektarios Georgalas. Pervasive and Mobile Computing. 2010 (2)

[3] Extending IP to Low-Power, Wireless Personal Area Networks [J]. Hui, Jonathan W, Culler, David E. IEEE Internet Computing. 2008 (4)

[4] Designing for other people’s strengths and motivations: Three cases using context, visions, and experiential prototypes [J]. P.J. Stappers, H. van Rijn, S.C. Kistemaker, A.E. Hennink, F. Sleeswijk Visser. Advanced Engineering Informatics. 2008 (2)

[5] Towards Mobile Ubiquitous Service Environment [J]. JI Yang, Zhang Ping, Hu Zheng, Wang Xu, Li Yinong, Tang Xiaosheng. Wireless Personal Communications. 2006 (1) 